\title{
Auszeichnung: Bibliotheks- und Informationszentrum Haßfurt als „Bibliothek des Jahres in kleinen Kommunen und Regionen 2021“ ausgezeichnet
}

https://doi.org/10.1515/bd-2022-0019

Der Deutsche Bibliotheksverband e.V. (dbv) und die Deutsche Telekom Stiftung haben am 23.10.2021 das Bibliotheks- und Informationszentrum Haßfurt (BIZ) als „Bibliothek des Jahres in kleinen Kommunen und Regionen 2021“ ausgezeichnet. Den mit 7.000 Euro dotierten Bibliothekspreis erhält das BIZ für seine vorbildliche regionale Vernetzungs- und Bildungsarbeit. In Kooperation mit mehreren kleinen Bibliotheken, Schulen, Kitas und einem Mehrgenerationenhaus erarbeitet das Bibliothekszentrum vielfältige Bildungsangebote für unterschiedliche Zielgruppen an verschiedenen Standorten.

Der Preis wurde von Dr. Frank Mentrup, Präsident des Deutschen Bibliotheksverbandes, sowie Dr. Ekkehard Winter, Geschäftsführer der Deutsche Telekom Stiftung, in Haßfurt überreicht.

Dr. Frank Mentrup, Präsident des Deutschen Bibliotheksverbandes hob die wichtige Rolle von Bibliotheken gerade in kleinen Kommunen und Regionen hervor: „Das BIZ Haßfurt hält mit seiner klugen und pragmatischen Arbeitsweise ein interessantes Angebot bereit, um die Menschen in seiner ländlich geprägten Region mit Medien, Informationen und Bildungsangeboten zu versorgen. $\mathrm{Ob}$ mit seinem Bücherlieferdienst in zwei Dorfläden der Region, mit seiner ,Bibliothek der Dinge‘, bei der Gegenstände wie Nähmaschinen, E-Book-Reader, ActionCams oder Laminiergeräte entliehen werden können, oder mit einer Multimediasäule mit zahlreichen Informationen für zugewanderte Menschen: Diese Arbeit stellt ein vorbildliches Modell bibliothekarischer Arbeit im ländlichen Raum dar."

Zur herausragenden Bildungsarbeit des BIZ ergänzte Dr. Ekkehard Winter, Geschäftsführer der Deutsche Telekom Stiftung: „Mit seinen Angeboten der Leseförderung, zu denen auch ein neu gegründeter Leseclub und ein Lesehund gehören, unterstützt das BIZ in vorbildlicher Weise Kinder und Jugendliche bei der Entwicklung ihrer Sprach- und Lesekompetenz. Die bereits mehrfach ausgezeichnete Schulbibliothek kooperiert systematisch mit Schulen und Kindergärten der Kommune und bietet den jeweiligen Klassenstufen angepasste und didaktisch aufbereitete Veranstaltungen an. Hier zeigt sich, wie sinnvoll und erfolgreich die 
Kooperation von schulischen und außerschulischen Bildungspartnern - gerade auch in ländlichen Regionen - sein kann.“

Annelie Ebert, Leiterin des Bibliotheks- und Informationszentrums Haßfurt bedankte sich im Namen aller Mitarbeiter*innen: „Wir haben uns über diese Auszeichnung unheimlich gefreut. Zumal wir damit nicht gerechnet haben. Es ist eine Würdigung unserer jahrelangen Arbeit und zugleich eine Anerkennung, stellvertretend erhalten für alle engagierten Bibliotheken im ländlichen Raum.“

Nähere Informationen zu der Preisträgerbibliothek unter: www.bibliotheks verband.de/bibliothek-des-jahres-kleinen-kommunen-und-regionen.

Am 24. Oktober 2021 wurde in Paderborn der Preis „Bibliothek des Jahres 2021“ verliehen. Diese Auszeichnung, die mit 20.000 Euro dotiert ist, erhielt die Stadtbibliothek Paderborn.

\section{Auszeichnung „Bibliothek des Jahres in kleinen Kommunen und Regionen“}

Im Jahr 2020 haben der Deutsche Bibliotheksverband e.V. (dbv) und die Deutsche Telekom Stiftung erstmals die Auszeichnung „Bibliothek des Jahres in kleinen Kommunen und Regionen“ verliehen. Der Preis würdigt Bibliotheken aller Sparten in Städten und Gemeinden mit bis zu 50.000 Einwohner^innen. Der Preis ist mit 7.000 Euro dotiert. Mit dem Preis wird vorbildliche und innovative Bibliotheksarbeit sowie Veranstaltungsarbeit, Bildungsangebote, Integrationsmaßnahmen sowie die Vermittlung von Informationskompetenz ausgezeichnet. Er soll einen Beitrag zum Image von Bibliotheken in kleinen Kommunen und Regionen leisten und der Motivation der Bibliotheken im Wettbewerb um Qualität und Innovation dienen.

Mehr Informationen unter: www.bibliotheksverband.de/bibliothek-desjahres-kleinen-kommunen-und-regionen

\section{Deutscher Bibliotheksverband}

Der Deutsche Bibliotheksverband e.V. (dbv) vertritt mit seinen fast 2.100 Mitgliedern bundesweit über 9.000 Bibliotheken mit 25.000 Beschäftigten und 11 Millionen Nutzer^innen. Sein zentrales Anliegen ist es, Bibliotheken zu stärken, damit sie allen Bürger^innen freien Zugang zu Informationen ermöglichen. Der Verband setzt sich ein für die Entwicklung innovativer Bibliotheksleistungen für Wissenschaft und Gesellschaft. Als politische Interessensvertretung unterstützt der dbv die Bibliotheken insbesondere auf den Feldern Informationskompetenz und Medienbildung, Leseförderung und bei der Ermöglichung kultureller und gesellschaftlicher Teilhabe für alle Bürger^innen.

URL: www.bibliotheksverband.de 


\section{Deutsche Telekom Stiftung}

Die Deutsche Telekom Stiftung wurde 2003 gegründet, um den Bildungs-, Forschungs- und Technologiestandort Deutschland zu stärken. Mit einem Kapital von 150 Millionen Euro gehört sie zu den großen Unternehmensstiftungen in Deutschland. Die Stiftung unterstützt gezielt Projekte, die sich an Kinder und Jugendliche im Alter von 10 bis 16 Jahren richten und sich mit Themen aus dem mathematisch-naturwissenschaftlich-technischen Umfeld beschäftigen.

URL: www.telekom-stiftung.de

\section{Pressekontakte}

Kristin Bäßler

Deutscher Bibliotheksverband

Leitung Kommunikation / Pressesprecherin

Tel.: + 49 (0)30 6449899 25 | E-Mail: baessler@bibliotheksverband.de

Matthias Adam

Deutsche Telekom Stiftung

Referent Kommunikation

Tel.: +49 (0)228 181920 15 | E-Mail: matthias.adam@telekom-stiftung.de

Verena Braun

Bibliotheks- und Informationszentrum Haßfurt

Tel.: +49 (0)9521951960 | E-Mail: verena.braun@biz-hassfurt.de 